\title{
BMJ Open Study protocol for a randomised controlled trial of a web-based behavioural lifestyle programme for emPOWERment in early Multiple Sclerosis (POWER@MS1)
}

To cite: Krause N, RiemannLorenz K, Steffen T, et al. Study protocol for a randomised controlled trial of a webbased behavioural lifestyle programme for emPOWERment in early Multiple Sclerosis (POWER@MS1). BMJ Open 2021;11:e041720. doi:10.1136/ bmjopen-2020-041720

- Prepublication history and additional materials for this paper are available online. To view these files, please visit the journal online (http://dx.doi. org/10.1136/bmjopen-2020041720).

Received 16 June 2020 Revised 17 December 2020 Accepted 14 January 2021

D Check for updates

(C) Author(s) (or their employer(s)) 2021. Re-use permitted under CC BY-NC. No commercial re-use. See rights and permissions. Published by BMJ.

For numbered affiliations see end of article.

Correspondence to

Nicole Krause; n.krause@uke.de

Nicole Krause (D) , ${ }^{1}$ Karin Riemann-Lorenz (D) , ${ }^{1}$ Tanja Steffen, ${ }^{1}$ Anne Christin Rahn (D) ,1,2 Jana Pöttgen (D) , 1,3 Jan-Patrick Stellmann (D) ,1,4 Sascha Köpke (1) , ${ }^{5}$ Tim Friede (D) , ${ }^{6}$ Andrea lcks (D) , ${ }^{7}$ Markus Vomhof (1) , Herbert Temmes, ${ }^{8}$ Markus van de Loo, ${ }^{8}$ Stefan M Gold (D) , 1,9 Christoph Heesen (D) ${ }^{1,3}$

\section{ABSTRACT}

Introduction Multiple sclerosis (MS) is an inflammatory and degenerative disease of the central nervous system that mainly affects young adults. Uncertainty is a major psychological burden of the disease from diagnosis to prognosis, enhanced by the pressure to make early decisions on a diverse set of immunotherapies. Watchful waiting for 1-2 years while adapting goals and lifestyle habits to life with a chronic disease represents another reasonable option for persons with MS (PwMS). A behaviour change programme based on evidence-based patient information (EBPI) is not available in standard care. This randomised controlled trial (RCT) with an embedded process evaluation investigates the efficacy and cost-effectiveness of a web-based behavioural lifestyle programme to change lifestyle behaviour and reduce inflammatory disease activity in PwMS.

Methods and analysis A web-based behavioural intervention will be evaluated in an RCT aiming to recruit 328 persons with clinically isolated syndrome, suspected MS or confirmed MS for less than 1 year, who have not yet started immunotherapy. Moreover, a mixed-methods process evaluation and a health economic evaluation will be carried out. Participants will be recruited in at least 16 MS centres across Germany and randomised to an intervention group with 12 months of access to EBPI about lifestyle factors in MS, combined with a complex behaviour change programme or to a control group (optimised standard care). The combined primary endpoint is the incidence of new T2 lesions on MRI or confirmed relapses.

Ethics and dissemination The study has been approved by the Ethics Committee of the Hamburg Chamber of Physicians (PV6015). Trial results will be communicated at scientific conferences and meetings and presented on relevant patient websites and in patient education seminars.

Trial registration number ClinicalTrials.gov Registry (NCT03968172); Pre-results.

\section{INTRODUCTION}

Multiple sclerosis (MS) is an inflammatory and degenerative disease of the central nervous
Strengths and limitations of this study

- Patients were actively involved in the development process of the intervention group programme in order to address the complex needs of persons with newly diagnosed multiple sclerosis.

- This study provides an opportunity to test if lifestyle interventions can influence surrogate measures of disease activity in an immune-mediated disease.

- The intervention does not include personal consultation, which may limit the extent and sustainability of changes in lifestyle habits.

- We aimed to design a patient-centred pragmatic trial and thus selected patient-reported outcomes as secondary endpoints; however, objective measures, as for example, accelerometry, are not included.

system that affects about 240000 people in Germany, typically first diagnosed during early adulthood. ${ }^{1}$ Over the past decade, new diagnostic criteria ${ }^{2}$ enabled earlier diagnosis of the disease and MRI has become a crucial diagnostic and prognostic instrument. Moreover, MRI is used for the evaluation of treatment success despite considerable limitations. ${ }^{3}$ However, there is still no highly specific diagnostic marker and diagnosis may remain unclear for years. In addition, reliable prognosis remains difficult and it is hardly possible to estimate the long-term expected disability, especially when based on disease development during the first 1-2 years after onset. For this reason, diagnostic information about MS is often experienced as traumatising and can cause disappointment and distrust in the medical system at an early stage. ${ }^{4}$ Although available immunotherapies reduce relapse 
rates, the long-term benefit on disability progression remains unclear. ${ }^{56}$ Nevertheless, early therapy directly after MS diagnosis is recommended. ${ }^{7}$ Adherence to immunotherapy in the first 2 years, however, may be as low as $30 \%-50 \%{ }^{8}$ These manifold uncertainties and the resulting psychological stress may have a negative effect on MS disease activity. ${ }^{9}$ Surveys have shown that persons with MS (PwMS) are a patient group that frequently uses internet sources to gather information. ${ }^{10}$ However, these sources often provide contradictory and poorly curated advice on lifestyle-related matters. ${ }^{11}$ The existing care structures cannot meet the complex information needs of PwMS. Experimental research as well as several clinical studies have suggested that improved lifestyle management may have the potential to impact inflammatory and neurodegenerative processes in MS. ${ }^{12}{ }^{13}$ Rigorous studies are largely missing and systematic, evidencebased patient information (EBPI) about lifestyle factors in MS combined with a behaviour change programme is not available. Training and empowerment interventions in MS have so far mainly been studied in face-to-face or group programmes. ${ }^{14}$ There are only very few examples for interventions that effectively change physical activity behaviour in MS. Motl et $a l^{15}$ have demonstrated in a pilot study that an internet-based intervention may change walking behaviour as assessed by self-report. However, online interventions in MS have mainly been investigated for the management of symptoms such as depression and fatigue, ${ }^{16}$ but not for change of overall lifestyle behaviour.

POWER@MS1 aims to encourage PwMS to find the best way of dealing with the disease on the basis of EBPI and a complex behaviour change intervention. The goal of the web-based behavioural lifestyle programme evaluated in this randomised controlled trial (RCT) is to optimise coping strategies and lifestyle habits, such as stress management, sleeping behaviour, physical activity and dietary behaviour. This may lead to decreased disease activity and lower distress to make an early treatment decision regarding use of immunotherapies. Together with the careful MRI monitoring of the disease dynamics in the study, this procedure might enable a more targeted immunotherapy initiation.

\section{Objectives}

This study investigates the hypothesis that EBPI about lifestyle factors in MS combined with a complex behaviour change programme (EBBC programme) can reduce inflammatory disease activity in MS and change patient behaviour.

\section{Primary objective}

To determine if the EBBC programme can reduce inflammatory disease activity in MS as measured clinically by relapses or by new T2 lesions on MRI.

\section{Secondary objectives}

The secondary objectives are to determine if the EBBC programme can:
- Strengthen patient autonomy and empowerment.

- Promote informed decisions on immunotherapy.

- Improve quality of life.

- Reduce anxiety and depression.

- Increase physical activity and a healthy dietary behaviour.

- Increase effectiveness of neurologist consultations.

- Fit with users and contextual factors.

- Save healthcare costs.

\section{METHODS AND ANALYSIS}

\section{Study design}

Based on developmental work following the Medical Research Council Framework for the development and evaluation of complex interventions, ${ }^{18}$ a web-based behavioural intervention programme on lifestyle adaptation in MS was developed (for details see the Interventions section). In addition, a web-based control group programme was developed based on information material available from the German Multiple Sclerosis Society (DMSG). Details with regard to the development and adaptation process will be reported in a separate publication.

The intervention will be evaluated in a superiority, rater-blinded, randomised controlled, parallel group trial. This protocol is focusing purely on the RCT. Study participants will be randomised to the intervention group (IG) with access to the EBBC programme in addition to standard of care or to the control group (CG) with optimised standard care using an allocation ratio of 1:1. In addition, a mixed-methods process evaluation (see online supplemental appendix 1) and a health economic evaluation will be carried out.

\section{Study setting}

Recruitment and neurological encounters will take place in community clinics, private practices and academic hospitals with a specialisation in MS across Germany.

\section{Eligibility criteria}

Persons aged between 18 and 65 years with clinically isolated syndrome, suspected or confirmed MS for less than 12 months, who signed informed consent, will be included. Furthermore, they must have at least two MS-typical lesions on T2-weighted images on MRI scans and an MS typical cerebrospinal fluid finding with detection of oligoclonal bands. Internet access is mandatory for participation. PwMS who are not able to provide informed consent or have a substantial psychiatric disorder or a substantial cognitive deficit based on clinical impression will be excluded. PwMS who have been treated with glatiramer acetate, teriflunomide, dimethylfumarate or interferons within the last 6 months prior to study inclusion or have received corticosteroid therapy within 4 weeks prior to study inclusion will also be excluded. PwMS with a planned treatment start within 3 months after inclusion or PwMS who had received any other MS-specific 
immunotherapy at any time in the past will not be eligible. Pregnancy and claustrophobia are also exclusion criteria.

\section{Interventions}

Eligible PwMS will be randomised to the IG programme or the CG programme. Both programmes will be offered online on the same platform with a similar design.

\section{IG: EBBC programme}

The IG programme is an MS-specific adaptation of the earlier developed 'Optimune ${ }^{\circledR}$ tool by GAIA (https:// gaia-group.com/en/). Based on current research and theory of the field, ${ }^{19-21}$ it was developed for lifestyle management in patients with cancer based on empowerment $^{22}$ and cognitive-behavioural therapy (CBT) approaches, including acceptance/mindfulness-oriented techniques. ${ }^{23-25}$ These techniques influence different theoretical domains as outlined in the theoretical domains framework $^{21}$ and thereby the participants' ability, motivation and opportunity to change their physical activity, stress management attitudes and dietary behaviour. For example, CBT techniques, such as behavioural activation and identifying and refuting unhelpful automatic thoughts and cognitive distortions, goal setting, goal review, agreeing on behavioural contracts, setting graded tasks, planning social support, action planning, weighing of pros and cons, preparing for/dealing with setbacks, self-motivational statements, constructing if-then plans, and formulating implementation intentions and positive emotion induction are incorporated throughout. Mental imagery exercises and mindfulness/acceptance exercises are integrated both in text format and as audio recording. Furthermore, EBPI, autonomy supportive intervention concepts based on self-determination theory, ${ }^{26}$ the principles of responsiveness ${ }^{27}$ and individual content-tailoring ${ }^{28}{ }^{29}$ are crucial components of the intervention format. The programme specifically attempts to avoid fear appeals and simple information provision (eg, 'lecturing'). The programme does not provide drugspecific information about available immunotherapies. The programme aims to translate evidence in the MS treatment and lifestyle management area in order to illustrate that decisions can be made. It follows the concept that every PwMS can develop an individual approach towards the disease, which might be a targeted immunotherapy initiation in one case or the development of a sophisticated food concept in the other.

The system is based on the Artificial Intelligence-based software platform broca ${ }^{\circledR}$, which is the basis for several effective therapy support systems evaluated in earlier RCTs $\left(\mathrm{eg},{ }^{16} 23\right.$ 30-32 $)$. An optional email and short message service reminder system (eg, with lifestyle-related stimuli or reminders regarding programme usage and newly activated modules) aim to enhance involvement. Usage of the IG programme will be monitored biweekly and reacted on after 4 weeks of non-usage to ensure patient adherence.
The programme is designed as a highly individualised system that provides PwMS with narrative and coordinated information based on their existing health beliefs, interests and so on. Each text passage ends with a set of preprogrammed response options in multiple-choice format reflecting possible reader's feedback, such as 'Yes, that makes sense' or 'I do not quite understand this yet'. The participant is invited to tick the matching response and will be guided to the next page referring to the choice, for example, 'I'm glad that you can understand it' or 'No problem. Then let me explain it in a little more detail'. These simulated dialogues lead to a highly individualised way through the intervention, while on the other hand, the programme makes sure that every important area is touched. More precisely, disease management and lifestyle techniques as well as exercises will be taught in sequentially activated interactive learning units ('simulated dialogues') focusing on the following topics:

1. Diagnosis, prognosis and immunotherapy decision-making.

2. Support in coping.

3. Techniques for coping with stress/depressive symptoms and developing positive emotions.

4. Optimisation of dietary behaviour.

5. Optimisation of physical activity behaviour.

6. Sleep hygiene and methods for dealing with insomnia.

The modules are not ordered by priority. Altogether, the IG programme consists of 16 modules and will accompany each participant over a period of 12 months with initial two to three weekly modules, later only weekly reminders and modules every 2 weeks, and four booster sessions at the end.

\section{CG: information from self-help societies}

CG participants will receive access to an information platform with optimised standard care consisting of information compiled from DMSG information material to reflect current practice. It will also accompany participants over a period of 12 months and cover similar topics as in the IG. A reminder function as well as usage monitoring and adherence promotion will be applied as in the IG.

\section{Patient and public involvement}

PwMS were involved in the development phase of the intervention and also participated in the feasibility and pilot testing of the IG programme (see the Study design section). They were given access to the programme and invited to evaluate content, practicability, userfriendliness and comprehensibility of the programme, also considering the needs of newly diagnosed PwMS. The programme was revised based on the acquired feedback (eg, technical adjustments, inclusion of more break possibilities and a progress bar in the modules). In addition, suggestions for prospective adjustments, which were not possible due to technical limitations, such as the embedding of video material, were gathered. Details regarding the feedback and resulting programme changes will be communicated in a separate publication. 


\section{Criteria for discontinuation and relevant concomitant care}

In case of new events (relapse or T2 lesion), formally the primary endpoint will be reached. However, study participants will be asked to remain in the study. Immunotherapy may be started during the trial period. Immunotherapy type, use and adherence rates will be collected during the clinical visits throughout the study.

\section{Outcomes}

Data will be collected over a period of 12 months, with a flexible follow-up of up to 24 months in early recruited PwMS. A list of outcomes, including measurement time points, is provided in table 1 .

\section{Primary outcome}

The primary endpoint is the time to a new relapse or, as a surrogate for inflammatory disease activity, a new lesion on T2-weighted images on MRI scans, whatever occurs first. Occurrence of new T2 lesions will be assessed according to an MRI protocol (Localizer, 3D fluid-attenuated inversion recovery sagittal, eg, $3 \times 3 \mathrm{~mm}$, 3D image T1-weighted native sagittal, $1-3 \mathrm{~mm}, \mathrm{PD} / \mathrm{T} 2$-weighted axial $3 \mathrm{~mm}$, protocol duration approximately $20 \mathrm{~min}$.). MRI scans will be read centrally by an experienced rater, blinded to subject identity and group assignment.

Relapses will be clinically evaluated by participating neurologists. In case of a relapse, duration of complaints/ impairment, relapse symptoms (worsened or newly occurred), degree of impairment due to the relapse and the degree of certainty with regard to the classification of the worsening as a relapse will be assessed.

\section{Secondary outcomes}

To assess risk knowledge, an abbreviated 10-item version of the MS risk knowledge questionnaire (Risk Knowledge in Relapsing Multiple Sclerosis V.2.0 $0^{33}$ ) will be used.

As a surrogate of decision quality, preferred and realised role preference in decision discussions for or against immunotherapy based on the Control Preference Scale ${ }^{34}$ will be assessed. Immunotherapy status will be assessed to determine whether an immunotherapy was newly started, aborted or changed.

The extent of patient activation (eg, expressed in the confidence and knowledge to take action as well as actually taking health-related action), based on the Patient Activation Measure ${ }^{35}$ and the coping capability, based on two items (items 10 and 24) of the Coping Self-efficacy Scale ${ }^{36}$ will be measured. In addition, patient expectancies based on items 1-3 of the credibility/expectancy questionnaire ${ }^{37}$ will be assessed. Based on principles of the Health Action Process Approach, ${ }^{38}$ readiness to change ${ }^{39}$ will be estimated in order to determine the interventions impact on willingness to change lifestyle habits. Moreover, changes in perceived empowerment (based on ${ }^{40}$, items 1, 3 and 4) will be measured.

Impairment in the Expanded Disability Status Scale ${ }^{41}$ will be determined by the treating neurologist.
Table 1 Assessments and measurement time points

\begin{tabular}{|c|c|c|c|c|c|c|c|c|c|}
\hline Instrument & Me & asu & rem & ent & time & poi & ints & & \\
\hline & $\mathrm{t}_{-1}$ & $t_{0}$ & $\mathrm{~V}_{1}$ & $\mathrm{~V}_{2}$ & $\mathrm{~V}_{3}$ & $\mathrm{~V}_{4}$ & $\mathrm{~V}_{5}^{*}$ & $\mathrm{~V}_{6}^{*}$ & $t_{x}$ \\
\hline Month & -1 & 0 & 1 & 3 & 6 & 12 & $18^{*}$ & $24^{*}$ & $x$ \\
\hline Eligibility screen & $x$ & & & & & & & & \\
\hline Informed consent & $x$ & & & & & & & & \\
\hline Demographic data & $x$ & & & & & & & & \\
\hline MRI & & $x$ & & $X$ & $x$ & $X$ & $\mathrm{X}$ & $x$ & \\
\hline Clinical visit & & $x$ & $x$ & $X$ & $\mathrm{X}$ & $\mathrm{X}$ & $x$ & X & $x$ \\
\hline Relapse history & & $x$ & $x$ & $\mathrm{X}$ & $x$ & $x$ & $\mathrm{X}$ & $x$ & $x$ \\
\hline $\begin{array}{l}\text { Immunotherapy } \\
\text { status }\end{array}$ & & $x$ & $x$ & 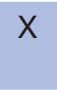 & $x$ & $X$ & $\mathrm{X}$ & X & $x$ \\
\hline EDSS & & $x$ & & & & $X$ & & & \\
\hline RIKNO & & & & $x$ & & & & & \\
\hline CPS & & & & & & $x$ & & & $x$ \\
\hline Decision satisfaction & & & & & & & & & $x$ \\
\hline Patient activation & & $x$ & & & & $X$ & & & \\
\hline Emotional coping & & $x$ & & & & $x$ & & & \\
\hline $\begin{array}{l}\text { Changes in } \\
\text { empowerment }\end{array}$ & & & & & & $x$ & & & \\
\hline Expectancy & & & $x$ & & & & & & \\
\hline Readiness to change & & $x$ & & $\mathrm{X}$ & & $X$ & & & \\
\hline HAQUAMS & & $x$ & & & & $X$ & & & \\
\hline EQ-5D-5L & & $x$ & & & $x$ & $X$ & $\mathrm{X}$ & $x$ & \\
\hline HADS & & $x$ & & & & $\mathrm{X}$ & & & \\
\hline GLTEQ & & $x$ & & & & $X$ & & & \\
\hline BSA & & $x$ & & & & $x$ & & & \\
\hline QHOD2 & & $x$ & & $X$ & & $X$ & & & \\
\hline myfood24 & & $x$ & & & & $x$ & & & \\
\hline Process evaluation & $x$ & $x$ & $x$ & $x$ & $x$ & $x$ & $x$ & $x$ & \\
\hline $\begin{array}{l}\text { Health economic } \\
\text { parameters }\end{array}$ & & $\mathrm{X}$ & & & $x$ & $X$ & $\mathrm{X}$ & $x$ & \\
\hline
\end{tabular}

$\mathrm{t}_{-1}=$ before enrolment; $\mathrm{t}_{0}=$ before allocation; $\mathrm{V}_{1}-\mathrm{V}_{6}=$ post allocation $\left(\mathrm{V}_{1}=\right.$ visit in month $1 ; \mathrm{V}_{2}=$ visit in month $3 ; \mathrm{V}_{3}=$ visit in month 6 ;

$\mathrm{V}_{4}=$ visit in month $12 ; \mathrm{V}_{5}=$ visit in month $18 ; \mathrm{V}_{6}=$ visit in month 24 ); $t_{x}=$ after reaching the primary endpoint.

${ }^{*}$ Only in early recruited PwMS.

BSA, Bewegungs- und Sportaktivität Fragebogen (Physical Activity, Exercise and Sport Questionnaire); CPS, Control Preference Scale; EDSS, Expanded Disability Status Scale; GLTEQ, Godin Leisure-Time Exercise Questionnaire; HADS, Hospital Anxiety and Depression Scale; HAQUAMS, Hamburg Quality of Life in Multiple Sclerosis Scale; PwMS, persons with multiple sclerosis; QHOD2, Questionnaire of Healthy Diet; RIKNO, Risk Knowledge in Relapsing Multiple Sclerosis.

Ideally, the lifestyle intervention leads to more general satisfaction with life but may also alleviate symptoms, such as depression, anxiety and fatigue. Quality of life will be measured with the Hamburg Quality of Life in MS Scale ${ }^{42}$ and the generic EQ-5D-5L. ${ }^{43}$ The Hospital Anxiety and Distress Scale ${ }^{44}$ will be used as a measure for depression and anxiety. 
Physical activity behaviour will be measured with the Godin Leisure-Time Exercise Questionnaire ${ }^{45}$ and the Physical Activity, Exercise and Sport Questionnaire (Bewegungs- und Sportaktivität Fragebogen). ${ }^{46}$

The Questionnaire of Healthy Diet, an adapted version of the Mediterranean Diet Screener as used in ${ }^{47}$ that was developed by the German Institute of Human Nutrition (DIfE), will be used to measure the frequency of intake of characteristic food groups within the last 7 days. To provide nutrient intake data, the 24-hour dietary recall myfood $24^{48}$ will be used, in each case three times within a time period of 1-3 weeks ( 2 weekdays, 1 weekend day).

\section{Health economic outcomes}

Health economic parameters will be assessed to determine the efficiency of the intervention by comparing the cost and outcome of the IG with the CG. All direct costs associated with the intervention as well as costs resulting from the consumption of health-related goods and services ${ }^{49}$ and indirect costs due to productivity losses will be considered from the perspective of the German statutory health insurance and the society.

To determine efficiency of the intervention, a costeffectiveness analysis will be performed in terms of additional costs per additional relapse or T2 lesion (clinical endpoint) averted and a cost-utility analysis, which aims to calculate the additional costs required for an additional improvement in quality-adjusted life years (QALYs). Incremental cost-effectiveness ratio and incremental costutility ratio will be calculated as the ratio of the difference in mean costs and difference in mean outcomes between IG and CG. QALYs will be measured by a wellestablished preference-based quality of life instrument (EQ-5D-5L) and evaluated by a German tariff to generate utilities. ${ }^{43} \mathrm{~A}$ standardised instrument ${ }^{50}$ will be used to record the healthcare consumption of study participants focusing mainly on outpatient doctor visits, visits to other health service providers, sick days, hospital stays and MS immune medication. Productivity losses will be estimated using the human capital approach. ${ }^{51}$ The $95 \%$ CIs for the outcome of the analyses will be determined nonparametrically based on the distribution characteristics of costs using bootstrap procedures. ${ }^{52}$ Univariate and probabilistic sensitivity analyses will be performed and costeffectiveness acceptance curves will be executed to take uncertainty into account. ${ }^{53}$

\section{Participant timeline}

The time schedule is depicted in figure 1.

\section{Sample size}

Based on effect sizes resulting from an RCT for a stress management intervention ${ }^{13}$ as well as data from cohorts on lesion development after an initial clinical event ( ${ }^{54}$ personal communication Michael Scheel, Charité Berlin), one event (relapse or at least one new T2 lesion) is expected in every second PwMS within 12 months in the CG. The 100 events result in a statistical power of $85 \%$ for

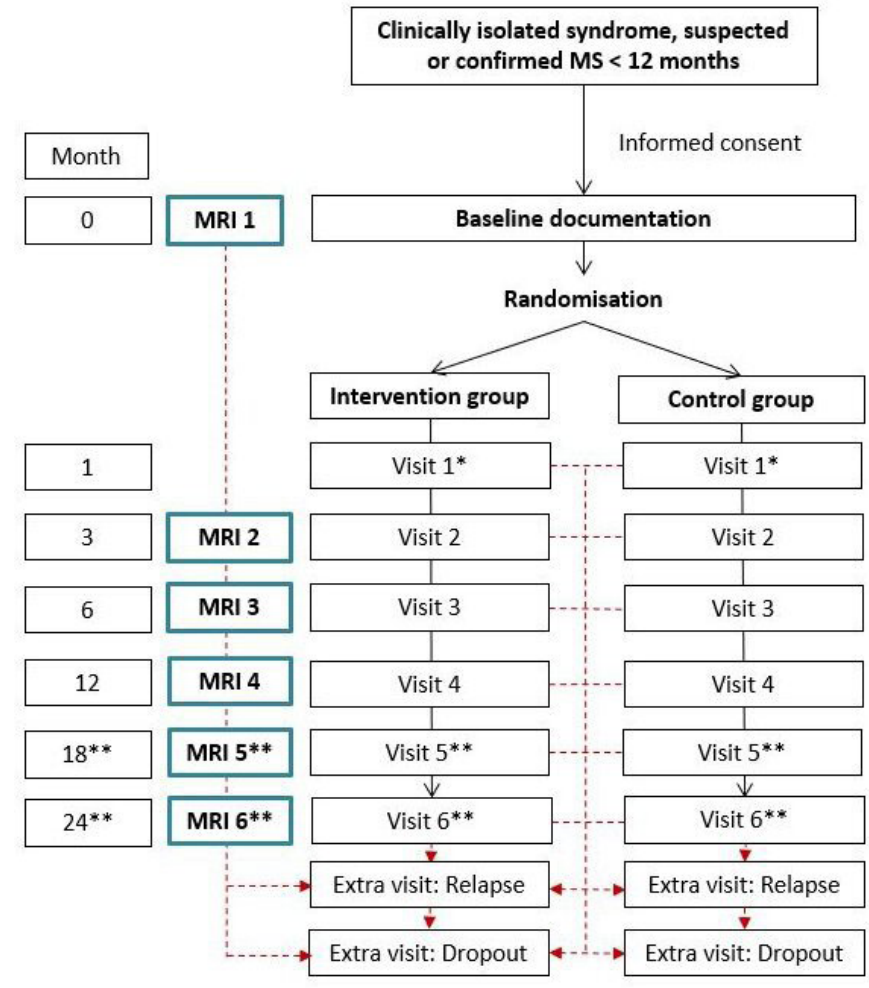

Figure 1 Participant timeline. *Visit 1 takes place in the MS centre to discuss the findings of the first MRI and by telephone through the study centre to clarify technical questions. ${ }^{* *}$ Visit 5 and visit 6 only in early recruited patients (flexible follow-up). MS, multiple sclerosis.

a two-way significance level test of $5 \%$ and an assumed HR of 0.55 , that is, a reduction of $45 \%$ by IG compared with the CG. Thus, with a mean observation time of 12 months, the 100 events required can be expected to be observed in 262 PwMS (131 per group). Assuming about 20\% dropouts over 1 year, 328 PwMS will be randomised (164 per group, $20 \%$ dropout $=33=131$ per group). A sample size recalculation will be performed after 12 months to review the assumptions on event rates and dropouts. ${ }^{55}$ If necessary, the number of cases will be increased to a maximum of 450 PwMS.

\section{Recruitment}

Eligible MS centres will be recruited by the coordinating centre in Hamburg (University Medical Center Hamburg-Eppendorf, UKE). Recruitment and inclusion of PwMS will take place in the participating MS centres through neurologists. In addition, POWER@MS1 will be advertised on the website of the DMSG. Overall, a recruitment period of 12 months is assumed with approximately 20 PwMS per centre, with one to two PwMS per month. Reasons for rejection will be documented.

\section{Allocation}

Group assignment will be undertaken externally and in a concealed manner through the electronic data capture system secuTrial ${ }^{\circledast}$ provided by the German MS Registry to prevent any manipulation of persons involved in the study. 
Eligible study participants will be randomised into the IG or to the CG in blocks (1:1 allocation ratio) through a computergenerated system in secuTrial ${ }^{\circledR}$. After baseline documentation and subsequent randomisation, PwMS will be provided with access (login) details to the IG or CG programme by an unblinded member of the study team.

\section{Blinding}

The study will be conducted as an investigator-blinded trial and participating MS centres will not be provided with any information about group assignment of a given PwMS. Blinding of the trial participants is pursued, but only possible to a limited extent. Participants and neurologists might realise their participation in the IG during encounters.

\section{Data collection methods}

Data will be obtained at different time points using paperbased and web-based questionnaires (see table 1). In case of missing data, participants will be contacted by a member of the UKE. All study relevant data will be entered into secuTrial $^{\circledR}$ and provided online. Results of MRI scans (image data) will be saved on compact discs (CDs). In accordance with current procedures implemented in medical practice, CDs with MRI data will be sent to the study centre in sealed envelopes via regular mail. This has been reviewed and accepted by the reviewing ethics committees and is in compliance with current data protection rules and regulations in Germany. They will be quality-checked, pseudonymised and uploaded in a protected reading centre database. Data obtained with regard to nutrition behaviour will be collected via secured online platforms of the Humanstudienzentrum of the DIfE and Dietary Assessment Limited (University of Leeds spinout company), which act in accordance with European Union General Data Protection Regulation (DatenschutzGrundverordnung). Data obtained through myfood24 will be stored on a server in the Netherlands, with a backup in the UK. After data collection, data will be transferred to secuTrial $^{\circledR}$ and connected with the existing data sets. In addition, usage of the web-based programmes will be monitored.

\section{Data management}

The IG and CG programme will be provided via a secure online platform that meets all legal requirements (SSL Encryption). All study data will be used and evaluated pseudonymously. However, all participating MS centres will have a list with names and assigned pseudonyms. All electronic and paper-based data material will be stored at the UKE for a maximum period of 10 years and will be destroyed subsequently. Stored CDs containing MRI images will be destroyed directly after analysis of the study data. In case of withdrawn consent, pseudonymised data will be anonymised. A deletion of already anonymised data is not possible.

\section{Statistical methods}

The effect on the primary endpoint will be estimated in a Cox proportional hazards regression that, in addition to treatment, also includes study centre as a factor; it will be reported as $\mathrm{HR}$ with $95 \% \mathrm{CI}$ and $\mathrm{p}$ value testing the null hypothesis
$\mathrm{H} 0: \mathrm{HR}=1$. Kaplan-Meier curves of the primary endpoint for both groups will be used to illustrate the treatment effect.

Secondary endpoints will be analysed using mean comparisons between IG and CG with adjustment for the baseline assessments and centre in analysis of covariance models. Least squares group differences will be reported with $95 \%$ CIs and $p$ values testing the null hypothesis of no intervention effect. The number of portions/day or week for different food groups will be analysed, evaluated and compared with current recommendations. Data obtained through the 24-hour recall (myfood24) will be used to analyse intake of selected nutrients of interest comparing mean changes in intake from baseline with post intervention between IG and CG, adjusting for baseline intake. MRI lesion counts will be analysed using negative binomial regression models, adjusting for baseline MRI and centre. Adverse events will be summarised as frequencies and percentages by treatment group.

In addition, subgroup and moderator variable analysis is planned to be performed (eg, early therapy vs no therapy and women vs men).

Reasons for study withdrawal will be reported. In case of missing data, all PwMS will be analysed in the group they were randomised to (intention-to-treat analysis). Early study discontinuations will be treated as independent right censoring in the primary analysis. In case of substantial or differential study discontinuations, the validity of the independent censoring assumption will be explored in shared random effects models of the primary endpoint and time to study discontinuation. To handle missing data in baseline variables or follow-up assessments, multiple imputation models will be applied.

All details of the statistical analyses including definitions of analysis populations will be prespecified in a statistical analysis plan.

\section{Monitoring}

As part of a risk-based quality management, external independent data monitoring including onsite visits at the UKE and remote data checks in secuTrial ${ }^{\circledR}$ will be performed by the contract research organisation CTC North GmbH \& Co.KG.

\section{Safety and adverse events}

As no significant harms (side effects, risks or complications) are to be expected, no stopping guidelines are planned. The performance of six MRIs over 2years is close to clinical standard and can be regarded as harmless. Contrast media will not be used in order to minimise the risk of possible contrast media deposition in the basal ganglia, although no information on depositions is available for the contrast media currently used. ${ }^{56}$ No auditing trials are planned or expected.

\section{ETHICS AND DISSEMINATION}

The study has been approved by the Ethics Committee of the Hamburg Chamber of Physicians (PV6015) and the ethics committees of participating study centres.

Informed consent (see online supplemental appendix 2) will be obtained by the participating MS centres and a 
copy will be sent to the study centre in Hamburg. Participants may withdraw their consent at any time. A financial compensation for participation in this study cannot be granted. In case of reaching the primary endpoint, PwMS are requested to remain in the study and continued access to the web tools will be guaranteed until the study end. Only the study team (investigators) and Alexander Stahmann (medical information scientist at the German MS Registry) will have access to the final trial data set. For publications, an anonymised data set will be used. If possible, an anonymised data set will be made available in the publication process in order to disseminate the study results.

Trial results will be communicated at scientific conferences and meetings (eg, at the yearly German Neurologists Society, the RIMS network) by the investigators and presented on the DMSG website and other relevant patient websites. Authorship will be shared between persons involved in the study following the current guidelines of the International Committee of Medical Journal Editors. Professional writers and persons not directly involved in the study will not be granted authorship.

\section{DISCUSSION}

This will be the first study assessing the impact of a lifestyle management programme combined with EBPI on inflammatory activity in MS. If successful, POWER@MS1 has a paradigm shifting potential. If successful, the trial could give lifestyle management a label as putative diseasemodifying. This can impact guideline development.

\section{Current trial status}

Recruitment of PwMS has started in July 2019.

\section{Author affiliations}

${ }^{1}$ Institute of Neuroimmunology and Multiple Sclerosis, University Medical Center Hamburg-Eppendorf, Hamburg, Germany

${ }^{2}$ Department of Health Services Research, Carl von Ossietzky University Oldenburg, Oldenburg, Germany

${ }^{3}$ Department of Neurology, University Medical Center Hamburg-Eppendorf, Hamburg, Germany

${ }^{4}$ APHM, Timone Hospital, CEMEREM, Marseille, France

${ }^{5}$ University of Cologne, Faculty of Medicine and University Hospital Cologe, Institute of Nursing Science, Cologne, Germany

${ }^{6}$ Department of Medical Statistics, University Medical Centre Göttingen, Göttingen, Germany

${ }^{7}$ Institute for Health Services Research and Health Economics, Centre for Health and Society, Faculty of Medicine, Heinrich Heine University Düsseldorf, Düsseldorf, Germany

${ }^{8}$ German Multiple Sclerosis Society, Federal Association, Hannover, Germany ${ }^{9}$ Department of Psychiatry and Medical Department, Section Psychosomatic Medicine, Charité-University Medicine Berlin, Berlin, Germany

\section{Twitter Tim Friede @tim friede}

Contributors $\mathrm{CH}$ is the principal investigator and led the planning and development of the full study with support from NK, KR-L, TS, ACR, JP, J-PS, SK, TF, SMG and HT. NK and CH wrote the first draft of the paper. TF specifically revised the statistical analyses sections of this paper. Al and MV provided health economic expertise. MvdL contributed as a PwMS expert. All authors conceived the study, revised the manuscript for relevant scientific content and approved the final version.
Funding This investigator-initiated study is publicly funded by the Innovationsfonds, Innovationsausschuss beim Gemeinsamen Bundesausschuss, Wegelystraße 8, 10623 Berlin, Germany (01VSF17015).

Disclaimer The funding body is not involved in any study-related aspect.

Competing interests $\mathrm{CH}$ has received research grants, speaker honoraria and travel grants from Biogen, Celgene, Genzyme, Merck, Roche. J-PS receives research funding from Deutsche Forschungsgemeinschaft and reports grants from Biogen and Genzyme outside the submitted work. TF reports personnel fees from Bayer, BiosenseWebster, Boehringer Ingelheim, CSL Behring, Daiichi Sankyo, Enanta, Fresenius Kabi, Galapagos, Immunic, Janssen, LivaNova, Novartis, Relaxera, Roche, and Vifor; all outside this work.

Patient consent for publication Not required.

Provenance and peer review Not commissioned; externally peer reviewed.

Supplemental material This content has been supplied by the author(s). It has not been vetted by BMJ Publishing Group Limited (BMJ) and may not have been peer-reviewed. Any opinions or recommendations discussed are solely those of the author(s) and are not endorsed by BMJ. BMJ disclaims all liability and responsibility arising from any reliance placed on the content. Where the content includes any translated material, BMJ does not warrant the accuracy and reliability of the translations (including but not limited to local regulations, clinical guidelines, terminology, drug names and drug dosages), and is not responsible for any error and/or omissions arising from translation and adaptation or otherwise.

Open access This is an open access article distributed in accordance with the Creative Commons Attribution Non Commercial (CC BY-NC 4.0) license, which permits others to distribute, remix, adapt, build upon this work non-commercially, and license their derivative works on different terms, provided the original work is properly cited, appropriate credit is given, any changes made indicated, and the use is non-commercial. See: http://creativecommons.org/licenses/by-nc/4.0/.

\section{ORCID iDs}

Nicole Krause http://orcid.org/0000-0001-6681-7054

Karin Riemann-Lorenz https://orcid.org/0000-0002-0779-2640

Anne Christin Rahn https://orcid.org/0000-0002-9051-3621

Jana Pöttgen https://orcid.org/0000-0002-6665-2154

Jan-Patrick Stellmann https://orcid.org/0000-0003-2565-2833

Sascha Köpke https://orcid.org/0000-0003-4106-4919

Tim Friede http://orcid.org/0000-0001-5347-7441

Andrea Icks https://orcid.org/0000-0002-4882-969X

Markus Vomhof https://orcid.org/0000-0002-2714-6371

Stefan M Gold https://orcid.org/0000-0001-5188-4799

Christoph Heesen https://orcid.org/0000-0001-8131-9467

\section{REFERENCES}

1 Petersen G, Wittmann R, Arndt V, et al. [Epidemiology of multiple sclerosis in Germany: regional differences and drug prescription in the claims data of the statutory health insurance]. Nervenarzt 2014;85:990-8.

2 Thompson AJ, Banwell BL, Barkhof F, et al. Diagnosis of multiple sclerosis: 2017 revisions of the McDonald criteria. Lancet Neurol 2018;17:162-73.

3 Rahn AC, Köpke S, Stellmann J-P, et al. Magnetic resonance imaging as a prognostic disability marker in clinically isolated syndrome: a systematic review. Acta Neurol Scand 2019;139:18-32.

4 Chalfant AM, Bryant RA, Fulcher G. Posttraumatic stress disorder following diagnosis of multiple sclerosis. J Trauma Stress 2004;17:423-8.

5 Tramacere I, Del Giovane C, Salanti G, et al. Immunomodulators and immunosuppressants for relapsing-remitting multiple sclerosis: a network meta-analysis. Cochrane Database Syst Rev 2015;9:CD011381.

6 Filippini G, Del Giovane C, Clerico M, et al. Treatment with diseasemodifying drugs for people with a first clinical attack suggestive of multiple sclerosis. Cochrane Database Syst Rev 2017;4:CD012200.

7 Gold R. Diagnose und therapie Der Multiplen Sklerose: Deutsche Gesellschaft für Neurologie, 2015. Available: https://www.dgn.org/ leitlinien/2333-II-31-2012-diagnose-und-therapie-der-multiplensklerose

8 Hansen K, Schüssel K, Kieble M, et al. Adherence to disease modifying drugs among patients with multiple sclerosis in Germany: a retrospective cohort study. PLoS One 2015;10:e0133279. 
9 Artemiadis AK, Anagnostouli MC, Alexopoulos EC. Stress as a risk factor for multiple sclerosis onset or relapse: a systematic review. Neuroepidemiology 2011;36:109-20.

10 Marrie RA, Leung S, Tyry T, et al. Use of eHealth and mHealth technology by persons with multiple sclerosis. Mult Scler Relat Disord 2019;27:13-19.

11 Beckett JM, Bird M-L, Pittaway JK, et al. Diet and multiple sclerosis: Scoping review of web-based recommendations. Interact $J$ Med Res 2019;8:e10050.

12 Ngandu T, Lehtisalo J, Solomon A, et al. A 2 year multidomain intervention of diet, exercise, cognitive training, and vascular risk monitoring versus control to prevent cognitive decline in at-risk elderly people (FINGER): a randomised controlled trial. Lancet 2015;385:2255-63.

13 Mohr DC, Lovera J, Brown T, et al. A randomized trial of stress management for the prevention of new brain lesions in MS. Neurology 2012;79:412-9.

14 Köpke S, Solari A, Rahn A, et al. Information provision for people with multiple sclerosis. Cochrane Database Syst Rev 2018;10:CD008757.

15 Motl RW, Hubbard EA, Bollaert RE. Randomized controlled trial of an e-learning designed behavioral intervention for increasing physical activity behavior in multiple sclerosis. Mult Scler J Exp Trans/ Clin 2017:3:205521731773488.

16 Fischer A, Schröder J, Vettorazzi E, et al. An online programme to reduce depression in patients with multiple sclerosis: a randomised controlled trial. Lancet Psychiatry 2015;2:217-23.

17 Pöttgen J, Lau S, Penner I, et al. Managing neuropsychological impairment in multiple sclerosis. Int J MS Care 2015;17:130-7.

18 Craig P, Dieppe P, Macintyre S, et al. Developing and evaluating complex interventions: the new medical Research Council guidance. Int J Nurs Stud 2013;50:587-92.

19 Michie S, van Stralen MM, West R. The behaviour change wheel: a new method for characterising and designing behaviour change interventions. Implement Sci 2011;6:42.

20 Michie S, Ashford S, Sniehotta FF, et al. A refined taxonomy of behaviour change techniques to help people change their physical activity and healthy eating behaviours: the CALO-RE taxonomy. Psychol Health 2011;26:1479-98.

21 Cane J, O'Connor D, Michie S. Validation of the theoretical domains framework for use in behaviour change and implementation research. Implement Sci 2012;7:37.

22 Werbrouck A, Swinnen E, Kerckhofs E, et al. How to empower patients? A systematic review and meta-analysis. Transl Behav Med 2018;8:660-74.

23 Twomey C, O'Reilly G, Meyer B. Effectiveness of an individuallytailored computerised CBT programme (Deprexis) for depression: a meta-analysis. Psychiatry Res 2017;256:371-7.

24 Samdal GB, Eide GE, Barth T, et al. Effective behaviour change techniques for physical activity and healthy eating in overweight and obese adults; systematic review and meta-regression analyses. Int $J$ Behav Nutr Phys Act 2017;14:42.

25 Cavanagh K, Strauss C, Forder L, et al. Can mindfulness and acceptance be learnt by self-help?: a systematic review and meta-analysis of mindfulness and acceptance-based self-help interventions. Clin Psychol Rev 2014:34:118-29.

26 Fortier MS, Duda JL, Guerin E, et al. Promoting physical activity: development and testing of self-determination theory-based interventions. Int J Behav Nutr Phys Act 2012;9:20.

27 Kramer U, Stiles WB. The responsiveness problem in psychotherapy: a review of proposed solutions. Clin Psychol Sci Prac 2015;22:277-95.

28 Krebs P, Prochaska JO, Rossi JS. A meta-analysis of computertailored interventions for health behavior change. Prev Med 2010:51:214-21.

29 Hawkins RP, Kreuter M, Resnicow K, et al. Understanding tailoring in communicating about health. Health Educ Res 2008;23:454-66.

30 Pöttgen J, Moss-Morris R, Wendebourg JM, et al. Online fatigue management program for patients with multiple sclerosis - a randomized controlled trial. Mult Scler 2015:21:41-2.

31 Zill JM, Meyer B, Topp J, et al. Vorvida: study protocol of a randomized controlled trial testing the effectiveness of internetbased self-help program for the reduction of alcohol consumption for adults. BMC Psychiatry 2016;16:19.

32 Berger T, Urech A, Krieger T, et al. Effects of a transdiagnostic unguided Internet intervention ('velibra') for anxiety disorders in primary care: results of a randomized controlled trial. Psychol Med 2017:47:67-80.
33 Heesen C, Pöttgen J, Rahn AC, et al. What should a person with relapsing-remitting multiple sclerosis know? - Focus group and survey data of a risk knowledge questionnaire (RIKNO 2.0). Mult Scler Relat Disord 2017:18:186-95.

34 Degner LF, Sloan JA, Venkatesh P. The control preferences scale. Can J Nurs Res 1997;29:21-43.

35 Hibbard JH, Stockard J, Mahoney ER, et al. Development of the patient activation measure (PAM): Conceptualizing and measuring activation in patients and consumers. Health Serv Res 2004;39:1005-26.

36 Chesney MA, Neilands TB, Chambers DB, et al. A validity and reliability study of the coping self-efficacy scale. $\mathrm{Br} \mathrm{J}$ Health Psycho 2006;11:421-37.

37 Devilly GJ, Borkovec TD. Psychometric properties of the credibility/expectancy questionnaire. J Behav Ther Exp Psychiatry 2000;31:73-86

38 Schwarzer R, Lippke S, Luszczynska A. Mechanisms of health behavior change in persons with chronic illness or disability: the health action process approach (HAPA). Rehabil Psychol 2011;56:161-70.

39 Lippke S, Ziegelmann JP, Schwarzer R, et al. Validity of stage assessment in the adoption and maintenance of physical activity and fruit and vegetable consumption. Health Psychol 2009;28:183-93.

40 Bann CM, Sirois FM, Walsh EG. Provider support in complementary and alternative medicine: exploring the role of patient empowerment. J Altern Complement Med 2010;16:745-52.

41 Kurtzke JF. Rating neurologic impairment in multiple sclerosis: an expanded disability status scale (EDSS). Neurology 1983;33:1444-52.

42 Gold SM, Heesen C, Schulz H, et al. Disease specific quality of life instruments in multiple sclerosis: validation of the Hamburg quality of life questionnaire in multiple sclerosis (HAQUAMS). Mult Scler 2001;7:119-30.

43 Herdman M, Gudex C, Lloyd A, et al. Development and preliminary testing of the new five-level version of EQ-5D (EQ-5D-5L). Qual Life Res 2011;20:1727-36.

44 Zigmond AS, Snaith RP. The hospital anxiety and depression scale. Acta Psychiatr Scand 1983;67:361-70.

45 Shephard R. Godin leisure-time exercise questionnaire. Med Sci Sports Exerc 1997;29:S36-8.

46 Fuchs R, Klaperski S, Gerber M, et al. Messung Der Bewegungsund Sportaktivität MIT dem BSA-Fragebogen. Zeitschrift für Gesundheitspsychologie 2015;23:60-76.

47 Estruch R, Ros E, Salas-Salvadó J, et al. Primary prevention of cardiovascular disease with a Mediterranean diet supplemented with extra-virgin olive oil or nuts. N Engl J Med 2018;378:e34.

48 Wark PA, Hardie LJ, Frost GS, et al. Validity of an online 24-h recall tool (myfood24) for dietary assessment in population studies: comparison with biomarkers and standard interviews. BMC Med 2018:16:136.

49 Krauth C, Hessel F, Hansmeier T, et al. [Empirical standard costs for health economic evaluation in Germany -- a proposal by the working group methods in health economic evaluation]. Gesundheitswesen 2005;67:736-46.

50 Chernyak N, Ernsting C, Icks A. Pre-test of questions on healthrelated resource use and expenditure, using behaviour coding and cognitive interviewing techniques. BMC Health Serv Res 2012;12:303.

51 Gold MR, Siegel JE, Russell LB, et al. Cost-effectiveness in health and medicine. New York: Oxford University Press, 1996.

52 Briggs AH, Wonderling DE, Mooney CZ. Pulling cost-effectiveness analysis up by its bootstraps: a non-parametric approach to confidence interval estimation. Health Econ 1997;6:327-40.

53 Fenwick E, O'Brien BJ, Briggs A. Cost-effectiveness acceptability curves-facts, fallacies and frequently asked questions. Health Econ 2004;13:405-15

54 Rosenkranz SC, Kaulen B, Neuhaus A, et al. Low clinical conversion rate in clinically isolated syndrome patients - diagnostic benefit of McDonald 2010 criteria? Eur J Neurol 2018;25:247-9.

55 Friede T, Pohlmann $\mathrm{H}$, Schmidli $\mathrm{H}$. Blinded sample size reestimation in event-driven clinical trials: methods and an application in multiple sclerosis. Pharm Stat 2019;18:351-65.

56 Costa L, Bracco P, Vada S, et al. A chemical analysis of the clogging process of polymeric biliary endoprostheses. Biomaterials $2001 ; 22 \cdot 3113-9$. 